\section{The Evolution of the Stars}

Drs. Hoyle and Lyttleton have raised ${ }^{\mathrm{I}}$ some objections to the results of the application of nuclear physics to the problems of stellar evolution as discussed in my recent articles on this subject ${ }^{2}$.

The main difference between the two points of view consists apparently in the fact that, whereas I maintain that the stellar mass remains essentially constant during the evolution, Hoyle and Lyttleton are of the opinion that the stellar energy, although manifestly due to nuclear reactions, actually comes from the cosmical material collected by stars on their way through space. It is easy to see, however, that the rates of accretion as calculated by Hoyle and Lyttleton ${ }^{3}$ are too high by a factor of many thousands, because they have not taken into account the thermal motion of the particles of the interstellar gas. This has led them to a formula according to which the amount of matter collected by a moving star is inversely proportional to the cube of its velocity. It is known, however, that, due to the interaction with the light quanta of interstellar radiation ${ }^{4}$, these particles must possess thermal velocities as high as $15 \mathrm{~km}$. per see. (for hydrogen). These high velocities reduce quite considerably the effective cross-section for capture, and the hydrogen collected can scarcely secure more than one twenty-thousandth part of the radiation of typical giants.

Also, I cannot agree with Hoyle and Lyttleton that "the basis of nuclear theory is in need of revision" in order to make possible the building-up processes in the stellar interior. Our present knowledge concerning the possible nuclear reactions is too well established (both experimentally and theoretically) to make any such revision possible. Thus, Weizsäcker's ${ }^{5}$ view that the elements are older than the stars remains the only way out.

The remark of Hoyle and Lyttleton that in my articles "no attempt is made to account for the three distinct periodicity groups of pulsating stars" is apparently due to the fact that they have overlooked the whole discussion pertaining to Fig. 2. In fact, the existence (disputed at present) of these three groups represents the best argument in favour of my views.

Finally, I quite agree with Messrs. Hoyle and Lyttleton that the problem of complex stellar groups (binaries and clusters) is still awaiting resolution.

George Washington University. G. Gamow. March 1

'Hoyle, F., and Lyttleton, R. A., NATURE, 44, 1019 (1939). 2 Gamow, G.; NatURe, 144, 575, 620 (1939).

3 Hoyle, F., and Lyttleton, R. A., Proc. Camb. Phil. Soc., (4), 35 (1939). - Dunham, T., Proc. Amer. Phil. Soc.. 8, 277 (1939).

${ }^{5}$ v. Weizsäcker, C., Phys. Z., 39, 633 (1938).

Prof. Gamow appears to agree with our view that accretion of hydrogen. by the stars from the cosmical cloud would be important if the rate were sufficiently rapid to compensate the transmutation of hydrogen within the stars. He believes, however, that the accretion rate is too low for this to be happening in the case of a typical giant by a factor of order $10^{4}$. Since the publication of our initial paper ${ }^{1}$ on this problem, we have been able to proceed further with the discussion of the physical aspects of the process, and we have been able to show that in reality the diff.culty mentioned by Prof. Gamow does not arise.
The accretion rate is proportional to $p$, the density of the cosmical cloud, and in our earlier papers we took an averaged value for $p$. But in certain regions of the galaxy, for example near the galactic plane, the density is probably much higher than the average for the galaxy as a whole. In addition, it has appeared from recent observational work ${ }^{2}$ that the average value of about $2 \times 10^{-23} \mathrm{gm}$. per c.c. at first taken by us may be too small by a factor of about 4 . Combining these effects, we have shown ${ }^{3}$ that the density probably exceeds $10^{-21} \mathrm{gm}$. per c.c. in exceptional regions of the galaxy, and the massive stars are regarded as being confined to these regions, thereby giving a natural explanation of such dynamical features as the marked concentration of massive stars towards the galactic plane. In this way a factor of $10^{2}$ is accounted for from the discrepancy urged by Prof. Gamow.

The remaining factor has arisen on account of the assumption by Prof. Gamow that the cosmical cloud everywhere possesses high thermal energy. It now appears from recent work $^{3}$ that in regions of density $10^{-21} \mathrm{gm}$. per c.c., the presence of even a small proportion of hydrogen molecules (of order 10 per cent, say) will prevent any appreciable temperature being maintained by the cloud. The reason for this is that the translatory energy of motion of the particles of the cloud must be in equilibrium with the internal energy of the molecules, since the thermal and internal energy tend to reach equipartition as a result of collisions within the cloud. Thus the effect of collisions is to excite the vibrational and rotational levels of the ${ }^{3} \Sigma$ state of the molecules, and this internal energy can be radiated by quadrupole transitions to the ground state of the molecules. In a cloud of density $10^{-21} \mathrm{gm}$. per c.c., the time between successive exciting collisions of a particular molecule is of order $10^{7}$ sec., whereas the lifetime for a quadrupole transition of the excited molecule is only about $10^{2} \mathrm{sec}$. Consequently the molecules radiate away the energy supplied by selective absorption, and it can readily be shown that the cloud will take up a temperature of approximately $6 \sqrt{T x / y}$, where $T$ is the temperature of the source of radiation illuminating the cloud, $x$ is the proportion of material in ionized form and $y$ is the proportion in molecular form.

For the purpose of applying this result to the accretion process, it is only necessary to show that an appreciable proportion of the cloud is in molecular form at a distance from the star of the order of the capture radius, since it must then follow that the material near the capture radius cannot rise to high temperature either by selective absorption or as a result of collisions caused by the gravitational field of the star. This question is of particular interest in relation to stars of high surface temperature, since these stars emit a relatively large quantity of radiation capable of disrupting the molecules. Consideration of this problem has shown that for such stars a molecule may persist at distances equal to the capture radius, as given by us for these stars ${ }^{1}$, provided the density of material through which the star is passing is sufficiently high. A value $10^{-21} \mathrm{gm}$. per c.c. for the density can be shown to be adequate for the main body of the $O$ and $B$ stars. In a cloud of this density the life-time against disruption by the action of stellar radiation is of order $10^{13}$ years. For a cloud of density $10^{-23} \mathrm{gm}$. per c.c. the life-time of the molecules is much shorter, and in large tracts of the galaxy it may well be the case that molecules are easily dissociated and constitute only a very small 